\title{
Literature Review: Picture Archiving and Communication Systems
}

\author{
Udo P. Schmiedl and Alan H. Rowberg
}

$\mathbf{T}$ HE LITERATURE ON Picture Archiving and Communication Systems (PACS) is very broad in its intrinsic nature and in the number and types of journals in which the publications may be found. Because most literature retrieval systems do not include search items specific enough to include PACS, searching must usually be done using broader terms such as "electronic imaging, digital imaging, radiology information systems, digital radiography, radiology department, hospital organization", and the literature searches that result contain a great deal of extraneous information. Only in $1990 \mathrm{did}$ the National Library of Medicine add "picture archiving and communication systems" to supplement their 1989 addition of "picture archiving, radiologic" with a cross reference listing. Unfortunately, these are both only cross references to the broader heading "Radiology Information Systems RIS," and one can search only on the broader terms or combine it with specific words in the title such as "picture" or "imaging."

In the support of research being performed at our institution, we performed a large number of literature searches and followed this with an extensive editing procedure, to remove articles that were not largely concerned with the issues related to PACS in a department of radiology. After the database was appropriately narrowed, each article was reviewed and abstracted. The abstracts were then dissassembled and sorted into subject matter areas, giving a narrative description of pertinent work in a variety of subject matter areas within PACS.

Unfortunately, this results in a snapshot of the literature at a certain point in time, and with the viewpoint of a single group of investigators. However, this document serves as a method of focusing one's individual research in a variety of areas, including educating clinicians about the

From the Department of Radiology, Imaging Research Laboratory RC-05, University of Washington, Seattle.

Address reprint requests to Alan $H$. Rowberg, $M D$, Department of Radiology, Imaging Research Laboratory RC-05, University of Washington, Seattle, WA 98195. (C) 1990 by W.B. Saunders Company. 0897-1889/90/0303-0016\$03.00/0 issues involved in PACS, support of graduate student teaching programs, training of radiology staff members, and support of clinical research projects.

\section{FUNCTIONAL STANDARDS}

\section{Acquisition Method}

Sonoda et al (1983) described a radiographic imaging system in which photostimulable phosphors are used as a temporary memory for the $\mathrm{x}$-ray image and is scanned by a laser. Since this publication, a number of digital radiographic imaging systems have been developed. One approach is line-scan radiography in which a slit collimator and a one dimensional detector array or an area type detector is employed. Using this technique, the scattered radiation is reduced significantly that increases in turn the contrast resolution. Based on this methodology, Doi et al (1986) described a digital radiographic system with a multiple slit scanning $\mathrm{x}$-ray beam that further reduces the scatter compared to a single slit, wide beam system. Great variations in transmitted x-ray intensity create serious problems in several radiographic applications, especially chest radiography. To circumvent some of these problems, patient specific beam attenuators for chest radiography as an alternative to scanned beam systems were designed (Hasegawa et al 1986). After a low-dose test exposure is digitized, a small cerium filter is printed and placed in the $\mathrm{x}$-ray beam near the collimator. The resulting images are analogous to optical or computer processed unsharp masking techniques and are typically uniformly exposed so that structures in all regions of the chest are simultaneously displayed with optimal film contrast. One obvious drawback of this approach is the potential spatial misregistration between time-separated dual exposures.

Dual energy subtraction imaging by a single $\mathrm{x}$-ray exposure are performed by using computed radiography with scanning laser-stimulated luminescence (Ishigaki et al, 1986). This method may be useful for spatial subtraction in chest radiography. In general images produced by digital radiography systems that utilize photostimulable 
phosphors are relatively uniform over a wide exposure range, virtually eliminating the need for repeats in portable radiography and other difficult examinations (Goodman et al, 1988). Worldwide, the number of scanning-laser-stimulated luminescence imaging plate systems (computed radiograph (CR) systems) in use has dramatically increased in Japan from 45 in 1986 to 250 in 1989. For comparison there are currently 35 units in use in Europe and 30 in the United States (Huang et al, 1990).

\section{Image Transmission to Workstation}

Image transmission via coaxial cables is likely to be available in most radiology departments throughout the country. They are, with a typical capacity of $10 \mathrm{Mbps}$ such as the Institute of Electrical and Electronic Engineers (IEEE) 802.3 Ethernet, inadequate for PACS image and text transfer systems (Martinez, 1989). A high-speed fiber optic network with rates over $200 \mathrm{Mbps}$ is required for integrated voice, data, and image communications (Martinez, 1989).

An IEE 802.6 distributed queueing dual buses/ metropolitan area network (MAN) standard as a network from file servers to medical workstations that simultaneously supports voice communication was proposed (Vallee, 1989). The system consists of optic fibers and comprises two unidirectional contra buses that can be joined to become looped buses. Data transmission up to $150 \mathrm{Mbps}$ per bus is possible. Two system configurations to perform radiological image transfer are considered. In the first system images were stored locally at the workstation using carrier sense multiple access collision detection as a local area network. Such a configuration minimizes the delay for display since congestion problems are avoided and the patients file can be preloaded to the local storage. The second configuration considered in this paper is a MAN (FDDI-II) network that combines the transfer of data packets and circuit switched packets (Vallee, 1989). It became evident that if the asynchronous bandwidth is not large enough to transfer the images, significant delay can occur. If the bandwidth is increased to $10 \mathrm{Mbps}$, the file server seems to be the bottle neck, again causing significant delays if the demand from several workstations for high resolution images during "image browsing" is high. A hierarchy of image storage levels, where the top level is the average of the image while the bottom level is the original is therefore suggested. A simulation study showed that the mean image display delay for each level of the pyramid is quite low for images up to $1024 \times 1024(0.5$ to $1.5 \mathrm{sec}$ ) (Vallee, 1989).

\section{Worklist Implementation}

The patient file has to be entered by a technician in the film library using an image entry workstation (Mastronadi et al, 1989). After having set up a folder for each patient, the technician proceeds to digitize, tag, and save the exam requisition sheet and each $x$-ray film. The image distribution process proceeds in background mode in parallel with subsequent image capture operations. This system obviously does not provide an interface that provides a certain level of intelligence to perform image transfer and storage automatically.

\section{PERFORMANCE STANDARDS}

\section{Time Requirements for Image Display}

Experience with the use of a PACS system for teleneuroradiology revealed that one of the major problems was the image display speed (Horri, 1989). It was felt that 26 seconds to fill a 20 image screen from the disk and 85 seconds to fill the same screen via fiber optic cable was too slow.

Investigating the working conventions and needs of radiologists it was found that the mounting and sorting of films was time consuming and irritating (Coristine et al, 1989). A top priority function of a PACS system was therefore intended to provide a practical solution to quickly setting up a case and allowing radiologists to sequence through a series of images.

\section{Image Display}

When an image is first displayed on the screen, its contrast should be automatically adjusted to provide an image of overall good quality.

To minimize image display time, a particular workstation described, maintains an 8 image memory backed up by a 30 image cache disk (Mastronadi et al, 1989). When an image is initially selected from the control screen, it takes 3.5 seconds until the image appears on the screen. If the image already resides in the file cache, it takes 1.5 seconds to redisplay the image. 
Reversed gray scale video display was found to be greatly inferior to conventional video display with respect to diagnostic accuracy in chest radiography (MacMahon et al, 1988).

The current practical experience shows that radiologists prefer hard copy to video display. The current 1,000 -line monitor can display the same dynamic range as the conventional radiograph but at a much lower light level and the display may not be linear. The video image is much easier degraded by ambient light. Random and structural noise due to the raster and electronic noise are more bothersome on a video display. Flicker and uneven resolution across the screen may further impair the quality of the video display (Goodman et al, 1988).

\section{Number of Images/Monitor: Image Files}

The optimal number of monitors (one, two, four, eight) is unknown and their geometric arrangement is often based on the attempt to emulate view boxes or alternators (Templeton et al, 1988).

To structure an image display system that connects three computed tomography (CT) scanners, four types of data folders are used (Becker et al, 1988): (1) the actual examination folder contains all slices of one examination plus the scout images, (2) the final examination folder is created when the physician has reported to the actual examination folder and contains all overview images $(128 \times 128)$ that are created from the full images and all full sized images, (3) the hospitalization report folder holds all final examination folders of one patient, and (4) the special folder is slice oriented and contains a collection of slices of different examinations for teaching and research purposes. Initially, the first overview images consisting of 16 token images $(128 \times 128)$ appear on the screen. By clicking a token image, the full sized image is displayed on one of the two other screens, where it can be viewed in detail. Alternatively, the physician is able to display $4256 \times 256$ images on one screen.

\section{Layout Arrangement}

Display and manipulation of image data is accomplished on network interactive diagnostic display station nodes that are a combination of gray-scale raster graphics hardware and software that implements a set of user friendly interactive display protocols detailing how the user and the graphics system are to interact (Templeton et al, 1988).

\section{Hard Copy in/output Options}

Since almost $80 \%$ of todays medical imaging procedures still employ traditional screen-film technology, an efficient method to convert x-ray films into digital data is essential. On the other hand, film will continue to play an important role in the radiology department even as PACS is being realized. Hard copy film is still the viewing medium preferred by the radiologist (Goodman et al, 1988). It is therefore necessary to implement hardware that reproduces digital images on film with high image fidelity. A laser scanner described digitizes a 14 inch $\times 17$ inches film to $2000 \times 2400 \times 10$ bit in 100 seconds at a speed of 229 lines per second (Lo et al, 1986). The laser printer can format 16 images on a 8 inches $x$ 10 inches or 14 inches $\times 17$ inches with up to $2300 \times 3050 \times 8$ bit resolution in 50 seconds. Both printer and scanner operate at a spatial resolution of 3 cycles per mm. Potentially such a system could contribute to the dose reduction for the patient by improving the contrast enhancement of the radiograph (Lo et al, 1986). It seems generally accepted that high-resolution (2 $\mathrm{K} \times 2.5 \mathrm{~K} \times 10$ to 12 bits) laser scanners are currently the best means for film digitization (Huang et al, 1989).

The performance of a linear array videocamera to a laser film digitizer for digital chest imaging was compared (Kundel et al, 1987). Test criterion was the ability of the film reader to classify pneumoconiosis. The linear array digitizer produced chest images with a spatial resolution of $1024 \times 1024$ pixels and 12 bits gray level in 2.4 minutes. Pixel size was $0.35 \mathrm{~mm}$. The laser scanner produced $1024 \times 1024$ images $(0.2 \mathrm{~mm}$ pixel size) and 12 bits depth in 60 seconds. The optical density range of the images was within the linear portions of both digitizers. The classification of profusion was impaired when images were digitized with the diode array camera but not when they were digitized with the laser scanner. The limitations of the images digitized with the camera were most likely due to limitations of the gray scale range and could be overcome with the use of a zoom function at the display station. 
A laser scanner that creates digital radiographs with a $2000 \times 2000$ pixel matrix (pixel size apprx. $0.2 \mathrm{~mm}$ ) was described (Sommer et al, 1985).

\section{Image Processing and Analysis}

An overview of basic concepts of image processing techniques in digital radiography has been presented (Barnes and Lauro, 1989).

A study investigating the impact of postprocessing on the detection of simulated pulmonary nodules with digital radiography showed that the specific algorithms such as moderate enhancement of medium frequencies, reversed gray scale images, reversed gray scale, enhancement of medium frequencies, and images with linear gradation curve and moderate enhancement of medium frequencies tested, did not improve the detection of pulmonary nodules. (Oestmann et al, 1989).

The possibilities of postprocessing and display of chest radiographs and mammograms including variable display of dynamic range, various gray scale options, image subtraction, and dual energy selective material cancellation was described (Sommer et al, 1984). The dual energy imaging capability seems promising with respect to characterization of tissue, including lung nodules, and useful for eliminating obscuring radiographic shadows in overlying regions of interest.

\section{Image Selection}

The function "previous image" was found useful, however, it was felt that it is more important to view two or more images simultaneously (Belanger et al, 1989).

\section{Saving Processed Images}

To redisplay an image at a later time exactly as it was seen last, the corresponding cache slot is modified (Mastronadi et al, 1989). This feature seems useful to prevent for example unnecessary readjustment of window and level settings, once the appropriate settings have been chosen by an individual radiologist for a particular study. The previous image can also be recalled without returning to the control screen (Mastronadi et al, 1989).

\section{Window/Level}

Reverse video image features were found particularly useful by chest and bone radiologists
(Belanger et al, 1989). Reverse images were found inferior for viewing chest radiographs (MacMahon et al, 1986). In another study more nodules were discovered on inverted images than with conventional image display (Sheline et al, 1987)

Image contrast can be adjusted in discrete steps or be inverted using the icon menu (Mastronadi et al, 1989). Fix window settings have been found sufficient and useful for reporting (Becker et al, 1988).

\section{Image Arithmetic}

A histogram-directed processing of digital chest images where the gray level threshold distinguishing mediastinum from lung fields was used to regionally selective process chest images was described (McAdams et al, 1986). This technique seems particularly useful for digital enhancement of underexposed mediastinal structures.

\section{Rotation}

The function "orientation" was found useful, but time consuming. For example, CT images should, if at all, be rotated $180^{\circ}$ at a time. The $90^{\circ}$ rotation was found superfluous (Belanger et al, 1989).

Image orientation can be adjusted using a series of right or left rotational and/or horizontal flips (Mastronadi et al, 1989).

\section{Filtering}

Edge enhancement using a spatial frequency filter clearly improves the diagnosis of pneumothorax (Goodman et al, 1988) and marginally improves the diagnosis of pulmonary nodules (MacMahon et al, 1986). In the case of pneumothorax, edge enhancement, and contrast manipulation can partly substitute for lesser spatial resolution (Goodman et al, 1988). Conversely, edge enhancement degrades the perception of interstitial disease.

\section{Zoom/Magnification}

The function of measurements and magnification were as noted from previous analog film studies found to be useful in $6 \%$ to $10 \%$ of cases (Belanger et al, 1989). 


\section{Reporting}

A radiological report may be organized as follows: a header that contains information related to the patient's examination such as the patient's name, exam title and date, the requisition order etc.; a body that contains a short history, diagnosis descriptions and conclusions and opinions; a signature which ratifies the content of the report. The report may be linked to the particular study in several ways once the report is generated. If the radiologist reads all the images and reports his findings, there is no direct link between the images and the description in the report and the report may be read by the referring clinician without reading the images. In this case, images may be stored separately from the report. If images and their description parts are mixed, the reading of the report is linked to reading the images by the referring physician and images are to be stored together with the reports. For both purposes, reports should consist of a generic logical structure as described above (Karmouch, 1989).

When a radiologist notices a new patient file in the action file, the images will have arrived at the workstation (Mastronadi et al, 1989). The images can be selected from a worklist and are transferred from the DIF in the local image store to the image screen for viewing. If a magnify operation is performed, a network request is issued to retrieve the appropriate original resolution segment from the OIF at the image entry workstation. The length of time it takes to retrieve the appropriate segment is directly proportional to its size. Once the images have been analyzed, the radiologist returns to the control screen and selects the "create report option." The system establishes a link between the workstation's voice terminal and a free channel on the voice mail system and a record/playback control panel appears on the screen. The new voice report remains private and can be edited or erased as long as the patients file is still open. As soon as the file is closed, the report becomes permanent, time stamped and can neither be deleted or modified in any way. After the report is completed and verified, the radiologist closes the patient's file and the system removes this patient's file from the radiologist's action file and sends it to the attending physicians action file with a status report that a radiology report is available (Mastronadi et al, 1989).

A speech recognizer with a 900 -word lexicon that was split into 5 sections by anatomic or subspecialty applications was described (Robbins et al, 1987). By switching from one lexicon to another and the use of trigger words, it is possible to dictate more than $70 \%$ of the reports. Dictation with voice entry takes about $20 \%$ longer than conventional dictation. Recognition reliability was found to be greater than $95 \%$. According to the authors, a voice recognition system integrated into software application of communication systems, is usable and cost effective. A text-free barcode report generation for mammographic examinations has been shown to, without increasing the radiologists workload, considerably reduce the dependency on transcription services (Adams et al, 1984).

Mamm Report is a report-coding system for mammography that runs on a microcomputer and speaks questions to the radiologist who responds by pressing on of two buttons on the computer mouse (Bramble et al, 1989). Coded reports are compressed at a ratio of $135: 1$ for computer storage.

\section{USABILITY STANDARDS}

\section{Nature of RIS/PACS Interface}

The design of two user interfaces for low cost radiological workstations is described (Gee et al, 1989). The first prototype interface RadUII uses pictorial directions to offer the user a quick overview over a single patient study in addition to a variety of image processing functions. This interface is connected to a single monitor display. The second user interfaces utilizes four independent, virtual display monitors on a single screen. In a modeling study with 12 radiologists using 12 patient CT studies, no significant advantage for either the single or the four image display configuration was found, although the four image display configuration was vastly preferred by the radiologists. The relatively small size of the patient studies may have been one reason that an equal number of radiologists responded for and against the utility of the pictorial directory, which may prove very helpful in the routine clinical use viewing studies with a large number of images.

An interface between a radiology information 
system (RIS) and PACS that is invisible to the user and is based on a one-way flow of data from the RIS to the PACS only was developed (Levine et al, 1989). The goal of the interface is to pass information between the PACS and the RIS in a timely efficient manner and to maintain a text data base to complement the images without having to enter patient data into the PACS system by hand. The one way interface may, however, be a less than ideal solution because delays in data acceptance, unmodified errors, and the necessity of dealing with multiple computer systems. A two-way interface might provide a more "intelligent" system that determines where information is needed and executes some of the operations currently performed by the users.

\section{Degree of Integration}

The user interface is extremely important for a clinically usable system (Taira et al, 1989). To evaluate the menu interface to the workstations, 11 first-time users were included and asked to perform 7 basic operations on only two simple menues that showed a directory of active patients and specific information of a chosen patient that provides the user with patient demographics and special processing or display features. The average time to display a specified case was 18.8 seconds, to display images from single anatomy 32.7 seconds, and to display earlier patient images was 50.3 seconds.

The American College of Radiology-National Electrical Manufacturer's Association (ACRNEMA) interface standard was designed to assure the exchange of information in medical imaging formats in a manner that does not rely on the use of custom designed interfaces for medical equipment (Wang et al, 1988). After thorough examination of existing standard interfaces, none was found to have enough advantages to be adopted as the ACR-NEMA standard. It is anticipated that the ACR-NEMA standard will be an evolving standard and several manufacturers are working on implementing this standard. In 1986, two prototype interfaces that implemented the standard were shown at the RSNA. A nontechnical description of the ACR-NEMA standard has been published (Spilker, 1989).

\section{HIS/PACS Interface}

A HIS (hospital information system)/PACS interface has been in operation at the University of Leiden since 1988 and facilitates the exchange of patient data, order data, and reports (Lodder $\mathrm{H}$ et al, 1988). The advantages as seen by the authors of having one communication system instead of two (HIS/RIS for administration and PACS for images) are (1) the clinical user can review all available patient data on one workstation and (2) the management of the great amounts of image data can be handled faster resulting in shorter waiting times that are more acceptable for the clinical user.

\section{Consultation}

When the attending physician notices a new patient file in the action file, the radiologist's report is ready and will appear on the control screen in a report list (Mastronadi et al, 1989). Report and/or images can be displayed and played back in any order. If the radiologist's comment (impression) does not seem sufficient to initiate treatment, the clinician can listen to the entire report on the voice mail system. After the patient file has been closed, it will automatically be removed from the clinician's action file and can only be reaccessed from the "other" patients list. If the clinician wishes to consult the radiologist while viewing a particular study, the system sends upon consultation request a snapshot of the patient file into a held patient file in the database. It further sends a tentative request to the radiologist's workstation and establishes a voice connection between the clinician and the radiologist. If the radiologist agrees to the consultation, his or her workstation is transformed into exactly the same state as the clinician's workstation. Also, both workstations are then synchronized in terms of mouse and keyboard input (Mastronadi et al, 1989).

The performance of a PACS workstation during scheduled conferences at peak image acquisition time to the performance of a film alternator was compared (Taira et al, 1989). For the film system an average of 34 seconds was required to access the next case of interest. For the digital system, the requirement to select the next patient and to display an average of 4.8 images was 21 seconds. For the film system, the average discussion time per patient was 55 seconds compared to 
75 seconds with the digital system. The image processing operations were found helpful in conveying the radiology diagnosis to the audience.

Unscheduled consultations over the phone or by clinicians walking over to the radiology department are ineffective and are expected to be substantially improved by the implementation of a PACS system (Taira et al, 1989).

\section{TECHNICAL CONSIDERATIONS}

\section{Workstation Characteristics}

Image presentation on the workstation (CommView) is being reported as still too slow and deficient for digital radiography or digital studies with a large number of changes. Image directories that zoom and leave through images at full resolution are still not available. Users should be able to compare, prefetch and synchronize image series (Mun et al, 1989). Workstations should have the necessary configuration to bring all necessary information together. Most importantly they should support an efficient way to generate radiology reports (Mun et al, 1989).

The ideal workstation is one that will mimic and possibly facilitate the direct consultation between two physicians by simultaneous transmission of image, voice, and text (Belanger et al, 1989). The workstation integrates the image display and the image control surface on one interactive screen (Belanger et al, 1989). Clinical tests showed that the system was easy to learn by radiologists resulting in few user errors.

A different user interface described consists of an interactive control screen (Macintosh Hypercard), a high-resolution image screen, and a voice terminal (Mastronadi et al, 1989). The user can move the mouse cursor freely between the image and the control screen minimizing the use of a keyboard.

\section{Network Characteristics}

The network for integrated PACS has to handle five different data groups: control data, knowledge data, image data, voice data, and image pointing overlay data. These data imply different transmission requirements to a network (Dwyer et al, 1988). The control, knowledge, and image overlay data require an error-free transfer, whereas voice and image data do not loose quality with single bit errors. For control and knowledge data, Ethernet and token ring proto- cols seem adequate (Martinez, 1989). A real time network transfers voice and image overlays at a high-speed using a token ring network. Image data are transferred using a high-speed circuit switched protocol. Three main channels allocated by time-division multiple-access scheme and high-speed voice, data and image traffic, fiber optic networks are desirable (Martinez, 1989). The importance of an integrated approach to data and image transmission is also stressed by others (Jost, 1989).

Modern multimode transmission systems operate on the basis of optic fiber technology and provide data transmission rates up to $200 \mathrm{Mbits} /$ second over distances up to $3 \mathrm{~km}$. As light sources, light emitting diodes seem to be more reliable and less expensive than lasers (Ricca, 1989). FDDI seems an emerging standard for optical local networks that is based on the IEEE 802.5 token ring standard. It is intended for the connection of high performance mainframes, workstations, and peripheral equipment and to provide a backbone connection for lower speed local area networks. Using the current FDDI architecture, several information packets can circulate at once with transfer rates for up to 80 Mbits per second. A star topology for the core zone in the radiology department is favored and expected to decrease in transmission time, for example, for a 14 inch $\times 17$ inch computed radiography image from 8 seconds to less than 1 second within the next 2 years using FDDI technology (Ricca, 1989).

The Integrated Services Digital Network (ISDN) is a worldwide public network standard that is intended to simultaneously provide end-toend digital communications of voice, and all types of packet switched and circuit switched data over the public switched network. ISDN might eliminate the need for independent, individual special purpose switched networks. ISDN also provides the user with a large number of services and flexibility. It seem obvious that ISDN will be competing with existing wide area network and MAN links for PACS communication purposes and will permit users access to remote PACS sites around the globe.

Although fiber optic links operate at very fast rates, the transfer time for computed radiography images is still too long to be clinically acceptable (Mun et al, 1989). The combination 
of fiber optic links, a T-1.5 link, a 19.2 dial up modem, and Ethernet have been proven useful (Mun et al, 1989). In particular, communication links that support data transmission at $0.5 \mathrm{Mbps}$ over a mile radius appear highly useful.

The following management functions of a digitally formatted radiology local network have been deducted (Cox et al, 1986): (1) acquiring and formatting data, (2) transmitting data, (3) displaying and manipulating data, (4) generating analog hard copies, and (5) archiving on-line and long-term. A model analysis conducted by the authors suggests that based on the 1986 technology, all digitally formatted images on a radiology network should be limited to $1024 \times$ $1024 \times 8$ bits. The required estimated throughput rate for an image management network may be $4 \mathrm{Mbps}$ with a signaling rate of $40 \mathrm{Mbps}$ (Cox et al, 1988). With a signaling rate of $40 \mathrm{Mbps}$ a typical examination consisting of $40512 \times$ $512 \times 16$ bit images would be transmitted across the network in 40 to 45 seconds. The throughput of the ETHERNET-Intel 8086/8087 prototype system was found to be too slow to use in a department wide image management system. A Hyperchannel-M68010 network can meet the minimum requirements for radiology image management given the current level of use of digital imaging (25\%) (Cox et al, 1988).

Two different $100 \mathrm{Mbps}$ local area networks are reported from Japan, which are one order of magnitude faster than Ethernet but are closed networks in the sense that only components from the same manufacturer can be connected to the network (Huang et al, 1989).

\section{Central Versus Distributed}

A central database usually allows for smooth retrieval of single cases by name or identification number, however, no search features may be available. The creation of image worklists is currently too slow to be used at image reading times. A central data management system that is connected to the image archive, the RIS through a PC, acquisition nodes, high-speed workstations located throughout the hospital, and a distant imaging center over a T-1 $1.5 \mathrm{Mbps}$ dedicated telephone line is described (Mun et al, 1989). The transmission medium used to connect the central node to key nodes is a $40 \mathrm{Mbps}$ fiber optic cable. Coaxial cables are used transmit text and image data to various oats of the system. The network topology is a star.

Questions as how the relationship between archive and other storage devices should be, and at what level of intelligence the data system should retrieve essential amounts of data, still remain open (Mun et al, 1989).

A centralized PACS system is characterized by a central host computer that manages multiple workstations as well as the image acquisition process (Cho et al, 1989). Advantages of this model are: (1) the resources are shareable, (2) analog image transmission using a video broadband network facilitates real time image transmission, high reliability, and does not need special software development, and (3) one patient directory can serve all the intensive care units and usage activities can be easily monitored. Disadvantages of this model are: (1) the resolution is limited to an image matrix of $512 \times 512$, (2) the workstation performance will be inconsistent and influenced by the workload of the central computer system, (3) when the central host computer is unavailable, all stations will become inoperable, and (4) due to a noisy electronic device, the image quality using a video broadband network may be degraded.

In the distributed model, each workstation consists of a microcomputer, image processor, magnetic disk, and display monitor (Cho et al, 1989). Advantages of this model are: (1) highresolution image display on the workstation $(1 \mathrm{~K}$ or higher), (2) distributed processing allows for steady local performance, (3) the use of a local area network permits flexible configuration of image transfer paths, and (4) each intensive care unit workstation is capable of standalone operations and, even during a temporary failure of the central host, remote workstations can continue to operate. Disadvantages of this model are necessary development of a network software and higher installation cost of additional workstations. Maintenance of the distributed model is more expensive because there are more hardware components to be maintained. Although the distributed model offers some major advantages over the centralized model, the latter may hold a place in interdepartmental image communication, especially if the cost issue is a critical factor (Cho et al, 1989). It has been predicted that PACS systems of the 1990s will utilize both 
centralized and distributed archives (Britt et al, 1989).

\section{ARCHIVE CHARACTERISTICS}

\section{Media}

Given the present technology, it is obvious that a PACS system will include optical disk technology as storage medium. At present, a large (12 inch to 14 inch) write once read many (WORM) disk seems most appropriate for image archiving. One or more jukeboxes will be configured with winchester magnetic disks for immediate buffering of archive/retrieve requests. This archive will be connected to the central PACS host computer or may be established as one or more file servers on high-speed workstation networks. Because of the 7 to 18 year requirement for image storage, any digital archive system must be able to accommodate new technologies as they emerge and be able to maintain existing archives (Mankovich et al, 1989).

Digital optical disks (12 inch) offer from 1 to 2.6 Gbytes of memory with data ranges from 2.0 to 5.0 megabits per sec (Hindel, 1986). Optical cards are nonerasable records that can be used to store medical records and have the advantage of high portability. Optical tapes can store up to 200 Gbytes on a 2400 foot long tape. X-ray archives that are accessed infrequently could be stored on tapes. Magnetic tape is the oldest storage medium and offers the largest capacity at the lowest cost. The drawbacks of this medium are the need for sequential accessing of data and the need to hand-mount the tape.

Flexible disks have typically a capacity of 300 kilobytes to 600 kilobytes, with experimental models extending the storage capacity to 10 Mbytes (Hindel, 1986). Flexible disks are generally not considered an archival medium of choice in a radiology department although they are used occasionally to store computed tomographic images. Nonremovable hard disks are often used in medical imaging because of their reliability and large storage capacity (600 Mbytes and over). Definite advantages of optical over magnetic storage media are that the latter are more susceptible to dust and scratches (Hindel, 1986).

\section{Storage levels}

Based on the ACR-NEMA standards, an image archive with a large memory (jukebox) in order to store images of 3 months to 6 months of hospital operation is proposed (Reijns, 1989). Images that will be studied the same or the following day are stored in a central buffer that contains a number of magnetic disks. Extended workstations and acquisition nodes are connected to the central buffer via its own memory buffer. A communication rate of $8 \mathrm{Mbps}$ seems sufficient to keep up satisfactory working conditions. The ACR-NEMA standard is based on 7 layers of order. At communication rates of $3 \mathrm{Mbps}$ the PACS requirements are met for a radiology department provided the multilayer system is used with uncompressed image data and sufficient local image memory (Reijns, 1989).

A published storage model for PACS classifies storage needs into three layers (Sherman, 1988). Layer 1 of storage contains active patient data that must be on line for fast access and consultation. These exams are subject to change until a diagnosis is made and approved; they are typically less than 1 week old. Currently, magnetic disks are preferred for layer one on account of their fast access. Layer 2, or mid term storage, stores exams for insurance and legal use and for historical medical consultations. Exams in this system are typically less than 2 years old. The storage medium of choice for this layer are large optical disks (WORM) in jukeboxes. When a patients exam is no longer accessed frequently, it is moved from layer 1 into layer 2 and erased from layer 1. Layer 3 is for long-term storage of patient data. If jukeboxes are used for layer 2 storage, then the storage medium for layer 3 data should consist of those optical disks that no longer reside in the jukeboxes. If a jukebox with erasable optical disks is used for layer 2 data, then layer 3 data should be backed up on optical tapes.

The storage medium currently preferred is a large diameter optical disk (Britt et al, 1989). The ACR-NEMA has developed an exchange medium standard for half inch 9-track magnetic tape but has not developed standard physical or logical requirements for optical disks as a storage medium. Consequently, no manufacturer's optical disks are logically compatible with any other's. It should be possible to only selectively dearchive a particular patient's head study without dearchiving the entire study of the patient (Britt et al, 1989). 
In an operational radiologic image archive on digital optical disks in a large university hospital, digital images are temporarily stored on magnetic disks and are archived onto WORM optical disks that are arranged in a mechanical jukebox at their full resolution (Mankovich et al, 1988). A pictorial index is maintained for each patient. The success rate for archival capture of images during the initial period was $96.6 \%$. Retrieval time for a 10 image series with 2,048 lines each image was 13 minutes. In the actual clinical setting, images are, however, rarely directly retrieved form optical disks. Rather if the system receives a request for a new study, the acquisition of the first image of this study initiates dearchival for all archived images of this particular patient that in turn caused overflow of the magnetic disk. A system that would facilitate selective dearchival of comparison studies of interest seems desirable.

\section{Capacity}

WORM technology currently mostly used by PACS systems with a diameter of 12 inch to 14 inch disks hold about 3 Gbytes per surface and sustain transferrates of $1.0 \mathrm{Mbps}$. Five and one quarter inch optical disks are currently available up to 400 Mbytes per surface with transfer rates up to $0.6 \mathrm{Mbps}$. The only accepted standard for 5 $1 / 4$-inch WORM is the cartridge form factor specified by the International Standards Organization. Jukeboxes are available for both large diameter and 5 1/4-inch optical disks. State of the art jukeboxes can hold 8912 -inch disks and can change disks in 8 seconds. The drives can access and transfer data concurrently (Britt et al, 1989).

Required storage space per 14 inch $\times 17$ inch film is approximately 6 Mbytes (Hedgcock MW, 1989).

Looking at storage requirements for a radiology department, only minor differences were found when comparing estimations based on film loads and exact image size. Estimations based on the case loads will produce figures that are $25 \%$ to $30 \%$ less than the actual yearly storage needs. If a film digitizer is used to convert standard radiographs to digital formats, the yearly storage capacity required will be $8 \%$ higher compared to CR. It is evident that for even a medium sized medical center jukeboxes with 6.8 Gbyte disks and effective compression techniques are required to fully implement PACS (Hedgcock, 1989).

The digital output in a radiology department (large teaching institution) is estimated at about 2,061 Gbytes per year (Jost et al, 1988). The rate of data flowing to the archive in a fully digital department averages 220 kilobytes per second. The flow to the archive is generally not a critical manner. Retrieval of images for physician review is a much more demanding problem. If a CT study is comprised of 7.4 Mbytes and should be delivered in less than 2 seconds, a throughput rate of $3.75 \mathrm{Mbps}$ will be required (Jost et al, 1988). If comparison studies are to be retrieved at the same speed, a much higher throughput rate will be required.

\section{Image Compression}

In a currently operational PACS system archive at a large hospital a reversible compression rate at 2:1 for digital images is used (Britt et al, 1989). Digital radiographs are compressed at a ratio $4: 1$. The higher ratio for film images is justified by the higher correlation between adjacent pixels since the image resolution is much higher, and by the effect of data packing on the 10-bit or 12-bit contrast data.

The effect of a quadtree-based data compression algorithm on the diagnostic yield in digitized radiographs was studied for 100 urograms at compression ratios ranging from 90:1 to $4.2: 1$ (Halpern et al, 1989). No loss of sensitivity, as measured by the number of findings, was found with a compression ratio of $4.2: 1$ compared to uncompressed images. Sensitivity decreased with increasing compression ratios at or above 11:1 and decreased more rapidly for calcifications than for soft-tissue masses.

\section{Compression Techniques}

Radiological images can be compressed either by the error-free or irreversible method. Errorfree image compression can yield an average compression rate of about $3: 1$, while irreversible image compression can produce a higher compression ratio. The image reconstructed by the errorfree method is identical to the original, while the image derived from the irreversibly compressed data set is somewhat different. A compression rate of $3: 1$ is, however, too low to efficiently 
increase image storage capacities. The full frame bit allocation technique (Shih-Chung et al, 1986) transforms the entire original image into the spatial frequency domain and produces a bitallocation table based on the characteristics of the transformed images and the desired compression ratio. The bit-allocation table is used to encode the transformed image and a onedimensional compressed image file is formed. The compressed-image file and bit-allocation table are used to reconstruct the image.

Compression ratios for digitized radiographs, $\mathrm{CR}$, and CT images were studied while each radiograph was digitized to $2048 \times 2048 \times 10$, $1024 \times 1024 \times 10$, and $512 \times 512 \times 10$ matrices using a laser digitizer (Shih-Chung et al, 1986). All images were compressed at five different ratios and in return reconstructed from the respective data sets. The image quality was quantified as the normalized mean square error (NMSE). A threshold NMSE of $0.02 \%$ specifies an image that has very subtle increase in noise that is very difficult to discern in a side-by-side comparison with the original. The acceptable compression ratio depends on the image matrix and the type of image. Chest radiographs digitized with a $1024 \times 1024$ matrix could be compressed with a ratio $30: 1$ and still maintained a NMSE under $0.02 \%$. In some cases, 2,048 matrix images can be compressed with an acceptable ratio as high as $30: 1$ or $40: 1$. Images with many edges such as pulmonary angiograms or barium studies always give lower compression ratios (20:1 for 1,024 matrix barium image) than for example chest films. Five hundred twelve matrix CT images could be compressed by 10:1 at acceptable image quality. The authors conclude that acceptable compression ratios for $2,048,1,024$, and 512 matrices are 25:1, 20:1, and 10:1, respectively.

\section{Archive Management/Availability of Radiographs/Scheduling}

In 1987, the mean time between completion of an exam and the approval of the diagnostic report was approximately 26 hours using a PACS/Comm View system (List et al, 1989). After a component of the CommView system called the results viewing station had been installed, the diagnostic cycle time was reduced to approximately 12.6 hours. The total time required to complete the dearchival and resequenc- ing of the exam films was shortened and the time that these films were out of the cycle had actually increased. Through development of local diagnostic image databases, the productivity of both the radiologist and referring physician is expected to further increase (List et al, 1989).

The average delay between acquisition and reporting was 20.1 hours for conventional methods of film handling in a pediatric hospital (Taira et al, 1989). For CR using a PACS system, the delay was 7.8 minutes. This difference was mainly due to the following reasons: (1) heavy film librarian workload, (2) unavailability of alternators, (3) film was taken to the clinical wards before radiology interpretation, and (4) impracticality of accumulating several films before hanging them on the alternator.

The folder concept is a potential method to approach data organization in PACS systems (Prior et al, 1989). If one defines a unique data object identifier, it is possible and advantageous to design a system architecture that includes distributed file servers and a centralized directory database server. The unique identification also permits the definition of a data object or a type folder that may be used to communicate association information between nodes. By expanding the folder concept to include database entities, it is possible to design a schema for a PACS database server that simulates the data organization currently used in radiology. By the use of both the folder as database entity and as data object, it is possible to deal with the potentially massive growth of PACS databases (Prior et al, 1989).

Patient scheduling often included as a module in modern information management systems can help avoid duplicate exams, facilitate reduction in patient waiting time, improved patient throughput, improved clinical history information, and improved billing accuracy and completeness (Arenson, 1988).

\section{Spatial Resolution}

For mammography, images digitized using a video camera and a $1024 \times 1024 \times 8$ image matrix were found not to be diagnostically suffcient (Binkhuysen, 1989). Using chest images, digitized with a laser digitizer and displayed at a resolution of $1280 \times 1024$, no difference in detectability of small nodules in patients with 
pneumoconiosis was found compared to the original images (Arenson, 1989).

A $512 \times 512$ resolution was found adequate for the majority of pediatric cases viewed by the clinicians (Taira et al, 1989). They were normally looking for relatively large structures such as heartsize, the presence of interstitial pulmonary fluid, signs of pulmonary infection, and placement of life support tubing. The $512 \times 512$ monitors were, however, not sufficient for the radiologist viewing more subtle findings such as small pulmonary edema and pneumothoraces.

Equal diagnostic accuracy for digital and screen film urography at pixel sizes of $0.2 \mathrm{~mm}$ to $0.33 \mathrm{~mm}$ was reported (Fajardo et al, 1989).

Image resolution of $3.3 \mathrm{lp}$ per $\mathrm{mm}(0.15 \mathrm{~mm}$ pixel size) of digital myelograms were found equal to $5 \mathrm{lp}$ per $\mathrm{mm}$ of conventional film myelograms (Yang et al, 1988).

\section{Scatter Reduction}

One of the major advantages of digital chest scanning systems is their optimal scatter reduction. Images acquired with point scanners are virtually free of scatter, whereas scatter still remains a problem to some degree with line scanning systems (Capp et al, 1985).

\section{Monitor Characteristics and Quality/Environmental Design}

Current commercial gray-scale cathode ray tube (CRT) monitors are capable of displaying $1280 \times 1024 \times 12$ bits. Few new, moderately expensive monitors are capable of displaying $2048 \times 1024 \times 12$ bit digital images (Templeton et al, 1988).

The importance of favourable environmental design for successful and ergonomical use of electronic imaging work stations is emphasized in the review by Horii et al (1989).

\section{Speed of Image Display}

Ideally, a CT study consisting of 7.4 Mbytes should be delivered in 2 seconds from the archive (Jost et al, 1988).

\section{Teleradiology}

The role of teleradiology on a large scale within a regional network of health facilities is presently under investigation. As it still seems unlikely that small community hospitals will be able to afford the cost and maintenance of expensive imaging equipment such as CT, magnetic resonance, DSA, or PET scanners it seems necessary to further design regional diagnostic centers providing services to several hospitals. These institutions could be connected to a tertiary care center via interactive telecommunication. These could provide rapid assessment of both invasive and noninvasive radiological studies at primary care facilities. (Carey, 1985).

Numerous teleradiology systems have been tested using telephone lines, ultrahigh frequency (UHF) radio, laser and microwave via line-ofsight towers and satellites. Telephone lines are practical because they are already in place in most areas. They allow transmission rates from 1,200-baud to $1.5 \mathrm{Mbits}$ per second, the average being 1,200 bits per second. The time to transmit a standard chest $\mathrm{x}$-ray over a standard telephone line is approximately 9.7 hours, not including coding. The time can be cut to approximately 0.5 hours by using four telephone lines. Under these conditions expenses for leasing the phone lines reach $\$ 4000$ a month (Carey, 1985).

An ultra-high-speed teleradiology system using integrated services digital network technology facilitates data transfer rates of up to 7,000 bytes per second and is expected to be expanded to rates up to 16,000 bytes per second via normal phone lines (Lear et al, 1989). At approximately $25 \%$ to $30 \%$ the speed of satellite data transmission this approach may offer an economical alternative to expensive satellite technology.

Broad band microwave transmission using either towers or satellites has been used successfully to transmit images. The cost of hardware required precludes, however, the exclusive use of this equipment for image transmission.

UHF transmission is satisfactory for short distances ( $<5$ miles), but with longer distances the image is degraded by interference from local radio stations, electronic equipment and humidity. Fiberoptic transmission is becoming more feasible as these systems become more widely available. This modality has the potential for multichannel digital broad band transmission at an enormous capacity (Carey, 1985).

\section{Clinical Experience with \\ Teleradiology Systems}

In 1962, a medical facility at Boston's Logan Airport was connected via microwave to the Massachusetts General Hospital over a distance 
of 2.7 miles. This system was used in 1970 to transmit chest $\mathrm{X}$-rays for remote interpretation. Agreement in interpretation using this system and the original chest $\mathrm{x}$-rays was found in $77 \%$ (Murphy, 1970; Carey, 1979).

In 1972, the use of slow scan television over existing phone lines to transmit images was investigated and found satisfactory for nuclear medicine studies. In 1973, teleradiology using UHF radio transmission with a band width of approximately 2.5 or $3 \mathrm{MHz}$ between two hospitals 10 miles apart was studied and again found satisfactory for nuclear medicine studies but less than satisfactory for radiographs (Carey, 1985).

A comparison between a closed-circuit television system with a microwave system and the conventional viewbox system showed that $78 \%$ of the decisions on the viewbox system, $75 \%$ of the decisions on the microwave system, and $65 \%$ of the decisions on the closed-circuit system were correct (Carey, 1985). Evaluation of a closedcircuit television link within a hospital using a high-resolution television camera, a coaxial cable and a standard television monitor showed that in $82 \%$ the correct diagnosis was made with this system, while the use of the viewbox yielded a correct diagnosis in $90 \%$ of the cases (Gayler, 1981).

In 1976 and 1977, the Hermes satellite was used to study possible benefits of interactive telecommunication within a regional health care system. Over a period of 5 months that this system provided teleradiological consultation, the service was considered 90 as efficient as direct film viewing (Page, 1982).

The slow-scan teleradiology trial using space technology for rural papgo advanced health care revealed that the reliability of the diagnosis was not acceptable. Increasing the perceived resolution from 500 to 1,000 line pairs per inch did not improve the diagnostic performance (Henceroth, 1977).

A study comparing telephone transmission of radiologic images in a closed-circuit system found that in $82 \%$ the cases were provisionally interpreted without discrepancy to the final reading. Using a teleradiology system with a $512 \times 512$ pixel format with 256 gray levels ( 8 bits) connected over telephone lines to the adjacent rooms results showed that the radiologist's scores for findings, impressions and confidence levels were consistently lower with this system than with the conventional viewbox (Heshiki, 1988).

Using the Canadian Satellite Anik-B to transmit radiologic images from Quebec to Montreal, a $93 \%$ agreement was found between television and direct interpretation. Comparing slow-scan television to broad-band teleradiology, it was found that the error rate on slow-scan television interpretation was twice as great as radiographic interpretation with the broad-band television system (Carey, 1985, 1979). In another trial using satellite image transmission, James et al found that video images of diagnostic quality could be transmitted via satellite, however not for all types of examinations. The diagnostic accuracy was $87 \%$ (James, 1982).

Comparing teleradiology systems from six manufacturers, all based on image transmission via telephone lines, no significant difference in the accuracy of the reading given directly from a $\mathrm{x}$-ray viewbox compared to the reports transmitted with the teleradiology system was found (White, 1989).

In a clinical trial of digital teleradiology in the practice of emergency room radiology, clinically significant discrepancies in 14 of the 897 cases (10 CTs, the rest radiographs) evaluated, were found (Kagetsu et al, 1987). The digital images using a matrix of $512 \times 512 \times 8$ were compressed at a 2.5:1 ratio and transmitted over a standard phone line performed worse than the conventional films in the diagnosis of fractures, pneumothorax, and calcifications (Kagetsu, 1987). The relatively long image transmission time (37.5 minutes for 12 CT images) caused a significant delay in the interpretation of emergency studies.

\section{Security/Legal Aspects}

Legal implications of storing a nonoriginal (compressed) image are not resolved although there is precedent in the common practice of printing and storing $C T$ and MR images as windowed pictures on film (Jost et al, 1988).

The primary aim of most workable, privileged access systems is not to exclude the appropriate and well-intentioned user of the system and to ensure the patient's right to confidentiality (James et al, 1986). The use of passwords may lead to inacceptable delays and sharing of this privileged information among other users may compromise the effectiveness of the system. Hierarchical 
access systems commonly used in business systems may prove to be so foreign to the present medical environment that they will be almost inacceptable. One might argue that the security problem is less serious in diagnostic modalities as compared to departments that deal with psychological or social problems. On the other hand, the patients clinical history is often essential for establishing the right diagnosis and this information would be available on an image management system and, therefore, accessible throughput the route of a less protected discipline. A dual data system is therefore suggested where a relatively secure system would be used to transmit data for an informed circle to establish the right diagnosis and a second system would be employed to transmit processed images.

\section{CLINICAL EFFICACY/EFFECTIVENESS}

\section{Head CT}

No significant difference in the detection of small infarcts seen on conventional and digitized (laser scanner $1024 \times 1024 \times 8$ ) head scans was found (Binkhuysen et al, 1989).

\section{Intensive Care}

The time interval between completion of the chest $\mathrm{x}$-ray acquisition and the primary viewing was decreased by 1 hour by the use of CR (Arenson, 1989). Films were often viewed by the intensive care unit physician before the radiologist.

\section{Pediatric Radiology}

Eighty two percent of radiographic images are acquired at $2048 \times 2048 \times 8$ bit resolution for the use of computed radiography in a pediatric hospital (Taira et al, 1989). The CR system outputs both a laser print hardcopy film and digitally formatted image data. The remaining $18 \%$ are films from outside hospitals. They are digitized using a laser scanner. Preliminary data indicate that there is no statistical difference in the diagnostic accuracy of conventional and computed radiography film images when detecting either pneumothoraces or solitary noncalcified lung nodules. The $x$-ray exposure delivered to the patient is reduced by $10 \%$ to $33 \%$ using CR.

\section{Chest}

Comparison of the diagnostic image quality of conventional films, and laser and videodigitized images that were obtained using a $3 \mathrm{M}$ chest phantom showed that the diagnostic accuracy was highest using conventional films (.95), followed by laser digitized images (.82), and videodigitized images (.74) (Winter et al, 1989). A much longer observation time was required to interpret digital images by the study group that consisted of radiologists that had no experience with the use of digital equipment (Winter et al, 1989).

The performance of a prototype dual-energy chest radiography unit (slit beam technique with sandwich detector) to a highly optimized conventional system was compared by using nodules of various sizes $(0.5 \mathrm{~cm}$ to $1.6 \mathrm{~cm})$ and calcium content superimposed on a human chest phantom (Niklason et al, 1986). Most likely due to the ability of the dual energy chest radiography unit to remove structured noise and the superior control of scatter, the detection of pulmonary nodules was far superior with this unit compared to conventional radiography. In a clinical study by the same group (Fraser et al, 1986), dual energy radiography was found to be highly accurate in assessing the presence or absence of calcifications in pulmonary nodules and thus determining their benignancy and malignancy.

The sensitivity of a low-dose flying spot radiography system for the chest was compared to conventional chest radiography (Kushner et al, 1987). Resolution of the prototype system was $512 \times 512 \times 10$ bit $(1.3 \mathrm{lp}$ per mm resolution) . The analysis of the double blind study of 120 patients showed that the film was more sensitive in the detection of pulmonary abnormalities. The digital images performed better in the detection of normal mediastinal and pleural soft tissue contours.

To assess whether it is possible to distinguish benign from malignant solitary pulmonary nodules with digital techniques, the chest $\mathrm{x}$-rays of 68 patients were digitized to $2048 \times 2048 \times 12$ bits and changes in the optical density within the nodules were analyzed. Striking differences were noted between 26 malignant nodules and 21 calcified granulomas. When applied to 21 benign nodules that had initially required thoracotomy, $43 \%$ percent were classified correctly (Sherrier et al, 1987).

Digitized chest films with finer resolution (2.5 lp per $\mathrm{mm}$, pixel size $0.2 \mathrm{~mm}$ ) ( $89 \%$ detection rate) and unsharp masking ( $1.25 \mathrm{lp}$ per $\mathrm{mm}, 85 \%$ 
detection rate) were superior to those with coarser resolution $(1.25 \mathrm{lp}$ per $\mathrm{mm}$ pixel size $0.4 \mathrm{~mm})$ (65\% detection rate) for the diagnosis of pneumothorax (Goodman et al, 1988). For normal patients no difference in diagnostic accuracy was found. For abnormalities other than pneumothorax, the unsharp mask images were significantly worse.

When nodules were projected over the lungs, a significantly greater number were identified on conventional radiographs than on radiographs acquired with a digital radiography unit (Chakraborty et al, 1986).

Comparing storage-phosphor digital bedside radiographs to standard radiograms in bedside imaging under phantom conditions, the digital radiograms were found to produce more consistent and ideal image densities than conventional radiography (Schaefer et al, 1989).

The detectability of pneumothoraces on computed radiographic chest images ( $2.5 \mathrm{lp}$ per $\mathrm{mm}$ ) was compared to their detectability on matched conventional screen film images ( $5 \mathrm{lp}$ per $\mathrm{mm}$ ). Among 8 radiologists, 4 performed worse when interpreting CR studies, independent of the size of the pneumothorax (Fajardo LL et al, 1989).

Evaluation of single exposure dual energy digital radiographs in the detection of pulmonary nodules and calcifications showed that digital "soft tissue" images were equivalent to conventional chest radiographs in detecting soft tissue pulmonary nodules. Digital "bone" images were significantly better in detecting calcified nodules than conventional radiographs (Oestmann et al, 1989).

\section{$G U$}

A computed radiographic system with conventional films for excretory urography was compared in 100 consecutive patients (Fajardo et al, 1989). A photostimulable bariumfluorohaldideeuropium imaging plate yielded a resolution of 2.5 to 5 line pairs per $\mathrm{mm}$ and a dynamic range that was 10 times greater than the dynamic range of conventional screen-film combinations. For scout abdomen films, the imaging plate sizes had a $1760 \times 2140$ matrix, $0.2 \mathrm{~mm}$ resolution, for scout tomograms, nephrotomograms and compression films a $1670 \times 2010$ matrix, $0.3 \mathrm{~mm}$ pixel size was used. The readout results of three radiologists were compared in 40 normal and 60 abnormal urograms. No significant difference with respect to sensitivity, specificity, or receiver operating characteristic curves were found between digital and screen film urograms. The readers' performances in making specific diagnoses were also the same with both techniques. Digital urograms that had pixel sizes of 0.2 to $0.33 \mathrm{~mm}$ obviously produced images that were diagnostically comparable to screen-film images. Postprocessing features of digital urography such as interactive contrast and density adjustments, filtering and edge enhancement were not evaluated in this study (Fajardo et al, 1989). Digital radiographs were generated at approximately half the exposure used for screen-film images without diminishing the diagnostic accuracy.

\section{Mammography}

Low diagnostic accuracy of digitized (video, and laser, both $1024 \times 1024 \times 8$ ) images compared to original mammograms was reported, because the pixel size was to large to detect small calcifications and slight blurring occured due to the large pixel size (Binkhuysen, 1989).

The detectability of microcalcification using xeromammography, digitized film mammography, and film mammography was studied (Smathers et al, 1986). The screen-fil mammograms were digitized with a laser film digitizer $(2048 \times 2048$ matrix) and displayed on CRT 525 line monitors. The radiologists used the following postprocessing features: level, window, and high and low bandpass filter. The 50\% detectability level for each technique was found to be as follows: xeromammography $0.55 \mathrm{~mm}$, digitized film $0.573 \mathrm{~mm}$, and screen film 0.661 $\mathrm{mm}$. The image processing capabilities of digitized films seem to improve the capability of screen film mammography to enhance the detection of microcalcifications.

Spatial resolution requirements and effect of unsharp mask filtering on the detectability of subtle microcalcifications were investigated (Chan et al, 1986). Digital images were created by digitizing conventional screen-film mammograms with a $0.1 \times 0.1 \mathrm{~mm}^{2}$ pixel size, processed with unsharp masking and then reproduced on film with a Fuji image processing system. Receiver-operating characteristic (ROC) analysis showed that digital mammograms with $0.1 \times 0.1$ $\mathrm{mm}^{2}$ pixel size provide lower detectability than the conventional screen-film mammograms. The 
detectability of microcalcifications was enhanced by unsharp masking.

The diagnostic potential of a PACS subsystem using videodigitized mammograms (1024x $1024 \times 8)$ and conventional mammograms was evaluated (Klessens et al, 1988). ROC analysis indicated a significantly worse performance of digitized films (0.67) compared to original mammograms ( 0.89 ). The discrepancy was most likely due to the long access time for digitized images and the difficulty to distinguish small film artifacts from small calcifications on digitized images.

\section{Musculoskeletal Radiology}

The value of digital radiography in disorders of large bones, joints, and muscles (excluding small bones and joints) was assessed (Pettersson et al, 1988). Conventional films were found superior in depicting small anatomic structures and delineating the zones close to prostheses. The frequency modified digital film $(1670 \times 2010$, $0.15 \mathrm{~mm}$ pixel size) was found superior in delineating soft tissues and areas with large attenuation differences.

Evaluation of radiation exposure of patients with scoliosis for digital radiography (flying spot technique, matrix $512 \times 512$ ) and conventional film screen radiography showed that the lower resolution digital radiograms were found to be of sufficient quality to perform scoliosis measurements and comparable within $1^{\circ}$ difference to film screen radiograms at many different angles of severity (Kushner et al, 1986). DR is therefore recommended for follow up studies in patients with scoliosis after tumors and structural abnormalities have been excluded on an initial radiogram.

A study on spatial resolution requirements for detection of subperiostal resorption demonstrated a significant improvement in diagnostic accuracy as pixel size decreases to the level of $0.08 \mathrm{~mm}$, whereas no further improvement was shown with pixel sizes of $0.04 \mathrm{~mm}(11.4 \mathrm{lp} / \mathrm{mm})$ (Murphey, 1989).

\section{Myelography}

A prospective study to compare the diagnostic efficacy of conventional film-screen imaging with $\mathrm{CR}$ in forty examinations that were done with both methods was performed (Yang et al, 1988). The digital images were acquired at a matrix size of $1670 \times 2010$ pixels, size $0.15 \mathrm{~mm}$ and a maximum resolution of $3.3 \mathrm{lp}$ per $\mathrm{mm}$. The resolution of the film-screen system was $5 \mathrm{lp}$ per $\mathrm{mm}$. The diagnostic accuracy of digital myelography was equal to that of conventional film-screen examinations. A slight preference for the conventional radiography with respect to penetration of the spinal column, visualization of the thecal sac and visualization of nerve roots and nerve root sleeves was noted that was ascribed to the reader familiarity with and confidence in conventional studies.

\section{GI}

The use of a $47 \mathrm{~cm}$ image intensifier for direct digital radiology was, at a $1024 \times 1024 \times 8$-bit resolution, successfully utilized for a variety of examinations, including barium and GU studies (Templeton et al, 1987). ROC analysis showed little statistical difference in the radiologists' response whether they were reading conventional chest radiographs or $1024 \times 1024$ laser printed digital radiographs.

\section{Dose Considerations}

Digital radiography (flying spot technique, matrix $512 \times 512$ ) required a skin exposure of only $2.4 \mathrm{mR}$ compared to $10 \mathrm{mR}$ at the chest and $60 \mathrm{mR}$ at the lumbar spine using film screen acquisition (Kushner et al, 1986).

Radiation exposure reductions of $96 \%$ to $98 \%$ were reported using CR compared to conventional radiography in radiographic leg length measurements in both children and adults (Kogutt, 1987).

Radiation exposure was reported to be reduced by $50 \%$ in study of intraoperative arteriography comparing phostimulable imaging plate radiographs with conventional screen-film due to decreased mAs levels (Pond et al, 1989). No difference in accuracy was found between the two imaging modalities.

\section{Spatial Resolution Requirements}

The minimum spatial resolution requirements for a total digital radiology department have not been defined. The spatial resolution requirements obviously differ among various imaging modalities and subspecialties. A $2.5 \mathrm{lp}$ per mm resolution that translates into a matrix of $2048 \times 2048$ for a 14 inch $\times 14$ inch chest film is required for pediatric chest films to facilitate the construction 
of a total digital radiology department possible with the present state-of-the-art technology (Seeley et al, 1987).

The minimum spatial resolution requirement for adult chest radiographs was $0.4 \mathrm{~mm}(1.25 \mathrm{lp}$ per $\mathrm{mm}$ ) (detection of septal lines) (Lams et al, 1986). For the detection of nodules (average diameter $12 \mathrm{~mm}$ ) the minimum spatial resolution was found to be $0.8 \mathrm{~mm}$ ( $0.625 \mathrm{lp}$ per $\mathrm{mm})$.

A study on the effect of pixel size on observer performance in the setting of subtle pulmonary abnormalities such as mild interstitial infiltrates and small pneumothoraces showed that diagnostic accuracy increases significantly as the pixel size is reduced to at least $0.1 \mathrm{~mm}(4056 \times 4056$ matrix for a $40 \mathrm{~cm} \times 40 \mathrm{~cm}$ chest film (MacMahon et al, 1986). The authors propose, as a compromise, a pixel size of $0.2 \mathrm{~mm}(2048 \times 2048)$ to be sufficient for digital radiography.

\section{Cost Effectiveness Considerations}

Although there are no empirical data as to whether a filmless system would be more economical than a conventional system for a medium to large sized hospital, forecasts predict an average annual costsaving of $\$ 840,000$ for a filmless department (Saarinen, 1989). A positive cash flow is predicted after 9 years if the acquisition costs of $\$ 7,500,000$ of a filmless system over a conventional system is taken into account. The cost of hardware to implement a radiology department image management network was estimated at approximately 2 to 3 million dollars by others (Templeton et al, 1988).

The diagnostic advantages demonstrated by digital systems to date do not yet justify their greatly added expense and complexity (Goodman et al, 1988). It is expected, however, as larger percentages of images will be obtained in digital format and as PACS systems will improve, the relative expense of digitizing conventional images will decrease.

\section{VARIA}

A digital radiology teaching library may be shared by several hospitals connected via PACS (Arenson, 1989). A medical image filing system as teaching files and found $1 \mathrm{~K} \times 1.5 \mathrm{~K}$ resolution satisfactory to display radiographs for the purpose of small groups of students (Kimura et al, 1989). A flexible retrieval scheme for diagnostic codes was found essential for the utility of the system.

A PACS system may have severe implications of the workstation for radiology practice. A survey conducted in the pediatric intensive care unit showed that the pediatricians felt that they could interpret $80 \%$ of the radiographs without the assistance of the radiologist (Taira et al, 1989). Only $26 \%$ of the written reports were read by the clinicians. A significant decrease of interaction between the clinician and the radiologist could result from the convenience of having radiographs readily available on the ward. On the other hand, serious consequences can arise from misinterpreted films that lead to unwarranted or improper medical treatment. The next generation of PACS systems should, therefore, incorporate the radiology reports as well as provide graphic annotation on suspect areas of radiographs to help increase the communication between referring physician and radiologist.

A study on the diagnostic accuracy of hard copy, conventional video, and reversed gray scale video display formats for chest radiograms showed that resolution was comparable with these modalities $(1024 \times 1024$ of hardcopies, 1,023-line video display monitor) (MacMahon et al, 1988). Diagnostic accuracy was found to be significantly greater with hard copy than with video display. The reversed gray scale video display yielded the lowest diagnostic accuracy. These individual differences as measured by ROC analysis, however, fell considerably short of statistical significance.

It still remains controversial as to whether a PACS system should be implemented in a modular or total fashion in a radiology department. Aspects of modular implementation for the coronary care unit and pediatric radiology are discussed by Huang et al (1990).

\section{REFERENCES}

Full reference citations for this article will appear in the November 1990 issue. 